\title{
CHEMICAL BIOLOGY BASED ON SMALL MOLECULE-PROTEIN INTERACTION
}

\author{
HIROYUKI OSADA
}

Chemical Biology Department, RIKEN Advanced Science Institute, Wako, Saitama, Japan

\subsection{INTRODUCTION}

Chemical biology is a new research field that applies chemistry to the investigation of biology and drug discovery. Bioactive organic compounds called bioprobes have been effective tools for elucidating the mechanism of many important cellular processes (Fig. 1-1). Such bioprobes are considered potential leads for the development of new therapeutics. One of the most important purposes of chemical biology is the search for small molecules that can control specific functions of proteins that will clarify currently unknown biological phenomena.

In this book new technologies are introduced for dissecting the interactions between small molecules and proteins and examples are provided of the identification of binding proteins of small molecules. Recent applications of small molecules as bioprobes to investigate biological systems are also described as

Protein Targeting with Small Molecules: Chemical Biology Techniques and Applications, Edited by Hiroyuki Osada

Copyright (C) 2009 John Wiley \& Sons, Inc. 


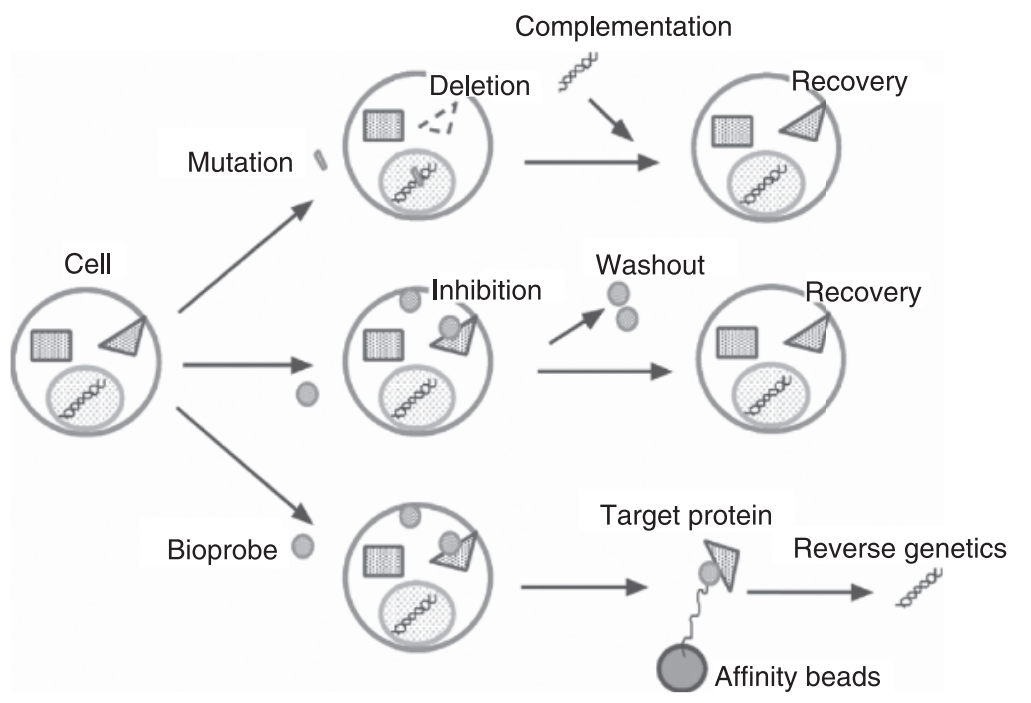

Figure 1-1 Bioprobes for chemical biology.

a typical topic of chemical biology. Chapter 12 provides a bird's-eye view of recent progress on small molecule-targeting proteins.

\subsection{BIOPROBES AS A TOOL OF CHEMICAL BIOLOGY}

The term bioprobe has been used by our group from the middle of 1990s to represent small organic compounds that are useful for investigating biological functions [1,2]. Until now, many bioactive small molecules that have shown antitumor, immunosuppressive, and other activities have been discovered from natural products or chemical libraries. Some of them are already in use as therapeutic medicines, but most of them have not yet been put into medicinal use. If the mechanism of action of a certain compound is revealed, the compound can be used for the investigation of biological functioning as a bioprobe. In general, when we are investigating a certain function of a protein, techniques of molecular biology, such as siRNA, are very useful. In the same way, an approach that uses a bioprobe to inhibit a certain function of a protein is also useful for dissecting a complex biological system. The approach that uses bioprobes instead of genetic tools, called chemical genetics, is a part of chemical biology. Much of the literature has reported that identification of a molecular target of small molecules is a successful strategy to use to reveal the biological functioning of a protein (see Chapters 2 to 11). Moreover, it is possible to identify the gene responsible for the protein by sequencing the protein captured by using affinity beads linked to bioprobes. 


\subsection{FROM CHEMICAL BIOLOGY TO CHEMICAL GENOMICS}

Originally, chemical biology was thought to dissect complex biological systems from the standpoint of organic chemistry. The nature of this field can be exemplified by the research that has been performed on the mode of action of two immunosuppressive agents (FK506 and cyclosporine); this research pioneered the field of chemical biology [3]. The immunosuppressive agents bind to the first target protein, called immunophilin (FK506 binding protein or cyclophilin A) [4]. At the beginning, inhibition of the enzymatic activity, peptidyl-prolyl cis-trans isomerase activity, which is carried by the immunophilin, is thought to be important. However, a later intensive study revealed that the complex of the immunosuppressive agent and immunophilin inhibited the final target, calcineurin [5]. In addition to the technique of molecular biology, the chemicals FK506 and cyclosporine A were used as bioprobes in these studies. The chemicals were used like an antibody to co-precipitate target proteins, which inspired the field of chemical biology [6].

Biochemistry reductively elucidates biological phenomena and describes biological phenomena at the chemical level. On the contrary, chemical biology starts at the chemical level and describes the big picture of biological phenomena. Molecular biologists often use the gene knockout technique and siRNA to investigate a particular protein function, and chemical biologists use chemicals or bioprobes instead. Simplicity, speed, and reversibility are the benefits of using bioprobes to induce the alteration of cellular phenotypes. There might be a problem if the specificity of the bioprobe is low, but it is very convenient to use chemicals instead of genetic tools. In contrast to genetic methods, the use of bioprobes does not require a complicated experimental procedure. When a bioprobe is added to a cell culture, the morphological alteration can be observed within a relatively short period. It is also possible to observe the reversal of the phenotype after washing out the chemical if the effect of the compound is reversible.

Whereas chemical biology is considered to be a basic science that focuses on a specific biological phenomenon, chemical genomics deals with genome-wide phenomena that are associated with informatics. Chemical genomics will enable rapid identification of novel drugs and drug targets. The goal of chemical genomics is to discover ligands (chemical probes) for all existing proteins. These ligands must be a very powerful tool for the study of proteins, cell functions, and biological processes that are relevant to physiology and disease.

\subsection{HOW TO EXPLORE SMALL MOLECULES}

Recent advancements in combinatorial chemistry have increased the number of organic compounds (small molecules) and given us new opportunities to use them in screening to develop new agents. Thus, pharmaceutical companies explore drug candidates from chemically synthesized compounds. It might be a rational approach to develop medicines that are applicable for oral administration. 
Because the molecular weight of natural products is usually larger than that of synthetic compounds, natural products are not considered to be suitable for medicines taken orally. However, it is not necessary to limit the screening source for chemical compounds when bioprobes are explored. Natural products vary widely in chemical structure as well as biological activity. Numerous compounds have been isolated from microbes, such as bacteria, actinomycetes, and fungi, and some are potential drug candidates.

Development of bioprobes starts from bioassay-based screening to explore the biological activities of a particular compound in extracts of microbial fermentation broth. After detecting sufficient activity, the active components are isolated by bioassay-guided purification. Physicochemical properties of the active components should be determined at the earliest possible stage and compared with those of known compounds to eliminate duplicate isolation. For novel compounds, sufficiently pure material should be obtained by large-scale fermentation of microorganisms to continue elucidation of the chemical structure and the evaluation of biological activity. Because the bioassay to detect desired compounds is very important, unique bioassays have been devised using intact cells or genetically engineered cells to express specific proteins. In the following sections, in addition to the conventional cell-based screening system, target-oriented high-throughput screening systems are described.

\subsubsection{Screening System Based on the Conventional Paper Disk Agar Method}

The paper disk agar plate method (halo assay) is the most conventional and commonly used bioassay system for antibiotics screening. Nowadays, this bioassay is applicable to genetically engineered microorganisms that express specific proteins or receptors as indicators. As an example, I would like to introduce the screening system that is used to detect antiviral compounds that are active against human immunodeficiency virus type I (HIV-1). HIV-1 is well known to be the causal virus of AIDS. However, it has not yet been clarified how HIV-1 infection induces $\mathrm{CD}^{+} \mathrm{T}$-cell depletion and eventual immunodeficiency in vivo. Cumulative evidence suggests that HIV-1 viral protein $\mathrm{R}$ (Vpr), one of the HIV-1-encoded proteins, may have an important role in its pathogenesis [7,8]. Until now, no clinical drug that targets Vpr has been developed; therefore, we have started a screen to isolate inhibitors of Vpr activity by using genetically engineered yeast. The growth of Saccharomyces cerevisiae, which carries the $v p r$ gene, is suppressed when Vpr protein is overexpressed. Using this indicator microorganism, we carried out a screen for a Vpr inhibitory compound from fungal metabolites. After many actinomycetes and fungal extracts were applied to this bioassay, we found inhibitory activity against Vpr in one fungal extract [9] and identified it as fumagillin, which already had been known as a potent inhibitor of angiogenesis [10]. Fumagillin not only suppressed the growth inhibitory activity of $\mathrm{Vpr}$ in yeast and human cells but also inhibited Vpr-dependent viral gene expression and HIV-1 virion production on viral infection of human macrophages. Our results demonstrate that fumagillin can be a lead compound for the development of a novel anti-AIDS drug that targets $\mathrm{Vpr}$ activity. 


\subsubsection{Screening Systems Based on Cellular Phenotypes}

During embryonic development, precursor cells differentiate into functional mature cells under the control of cytokines and hormones. For example, tumor cells, including leukemia and neuroblastoma, are thought to forfeit differentiation programs. Osteoporosis is explained simply as the hyperactivation of osteoclasts rather than osteoblasts. Osteoclasts are bone-resorptive multinucleated cells that are derived from hematopoietic stem cells of the monocyte/macrophage lineage. Osteoclasts are involved in remodeling bone matrix in concert with osteoblasts/stromal cells. Osteoclasts are differentiated from precursor cells by the aid of two critical factors: receptor activator of NF- $\kappa$ B ligand (RANKL) and macrophage colony-stimulating factor (M-CSF), which are supplied by osteoblasts [11].

Because the abnormal enhancement of osteoclasts is implicated in a variety of human diseases, including osteoporosis, rheumatoid arthritis, and cancer bone metastasis, it is worthwhile controlling osteoclast differentiation by small molecules [12]. Osteoclastogenesis inhibitors not only would be useful as tools in osteoclast biology but can also be developed as therapeutic drugs for these bone-related diseases. To identify small molecules that inhibit osteoclast differentiation, we have performed cellular phenotype-based assays. In the course of screening we found gerfelin and methylgerfelin [13] as active compounds against osteoclastogenesis. Gerfelin and methylgerfelin were revealed to be inhibitors of osteoclastogenesis through the inhibition of glyoxalase 1 activity [13].

\subsubsection{Screening System Based on Physical Interaction}

Microbial metabolites contain a wide variety of compounds that regulate cell proliferation through the specific inhibition of key proteins that are required for cell proliferation. Although it is possible to find such a cell growth inhibitor by the direct observation of cell growth, it is also possible to find cell-cycle inhibitors by a screening method that is based on protein-protein interactions, which is a method similar to the receptor binding assay [14]. A fluorescently labeled ligand is synthesized and applied to the target receptor/protein, which is immobilized on the surface of a 96-well plate. The binding between the fluorescently labeled ligand and the receptor is measured by a fluorometer. This assay system is suitable for high-throughput screening and is applicable to various types of screening for protein-protein interaction inhibitors.

As an example, I will describe the screening of polo-like kinase 1 (Plk1) inhibitors. Plk1 is one of the key regulators of mitotic cell division [15]. Localization of Plk1 changes dramatically in the M phase. During late G2 and prophase, Plk1 localizes to the centrosomes. At metaphase, Plk1 localizes to the kinetochores, where it is involved in the regulation of spindle assembly and chromosome segregation. In anaphase, Plk1 is found at the center of the spindle, and in telophase, Plk1 is found on the contractile ring. The C-terminal polo-box domain (PBD) is involved in the localization of Plk1. PBD was recently revealed to be a phosphopeptide-binding domain in various important proteins that are required 
for cell-cycle regulation. PBD-dependent binding is not only important for the subcellular localization of Plk1 but is also necessary for targeting of the protein to specific substrates. Therefore, we have established a screening system to find inhibitors of PBD-dependent binding in a high-throughput manner [16]. To quantify binding, fluorescent fusion proteins (which contain monomeric Venus and PBD) were expressed in Escherichia coli. Phosphopeptides of a PBD-binding sequence that was derived from human Wee1A were chemically synthesized and bound covalently to 96 -well plates. The fusion protein was mixed with candidate compounds and placed into each well. The unbound PBD was washed out, and bound PBD was quantified by a spectrofluorometer. When a binding inhibitor of PBD is present in the assay system, it is expected that the fluorescence level decreases. Among about 2500 compounds that were tested, purpurogallin (PPG), a benzotropolone derivative originally isolated from nutgalls [17], was identified as the most potent inhibitor of PBD-dependent binding. Interestingly, however, compounds similar to PPG that lacked a hydroxyl group did not have inhibitory activity, indicating that the hydroxyl group of PPG is essential for the inhibitory activity. PPG had previously been reported as an inhibitor of the interaction of the Bcl-XL and BAD proteins [18]. However, the inhibitory concentration of PPG against Bcl-XL and BAD was higher than that against Plk1. Therefore, it is thought that the action of PPG is rather specific to the PBD-Wee1 interaction. We then examined the role of PBD-dependent binding on the progression of mitosis using purpurogallin. Previous studies showed that inhibition of Plk1 kinase activity induces prometaphase arrest with abnormal spindles, leading to the observation of monopolar cells. However, monopolar spindle cells were not observed in PPG-treated cells. Mitotic spindles in PPG-treated cells apparently were normal, but careful examination after staining with anti $\alpha$ - and $\gamma$-tubulin antibodies revealed that the spindle poles in PPG-treated cells were diffuse and more distanced than those of the control cells. The majority of chromosomes in the cells aligned at the metaphase plate, but some chromosomes were unaligned and located close to the spindle poles. This phenotype is similar to that of PBD overexpression in the cell.

\subsection{PROFILING: A POWERFUL STRATEGY FOR TARGET SPECULATION}

When bioactive small molecules are discovered by cell-based screening, its molecular target must be elucidated. If a compound has the possibility of becoming a drug, potential targets must be defined carefully to predict the side effects. To know the molecular target, we have to examine a lot of possible targets. In some cases, there may be unknown molecular targets. For these reasons, the identification of molecular targets for small molecules is usually a difficult and time-consuming process. When there are many possible target candidates for a small molecule, we have to narrow the range of possible targets. There have been several reports on the identification of binding proteins, such as a direct method 
that uses affinity matrices that arm small molecules to pull down binding proteins [19] and profiling of the phenotypes of small molecules [20]. Comparison of cell growth inhibitory concentrations of small molecules against various cell lines is informative in predicting the target of a small molecule. Using their profiling method, which is based on the drug sensitivity of cancer cells, Yaguchi et al. identified the molecular target of ZSTK474 to be phosphatidylinositol 3-kinase [21]. Comprehensive analyses that integrate bioinformatics are suitable and may be more informative for this purpose.

Biologically active small molecules affect cellular processes, induce modification of their target proteins, and change protein expression levels. When cells are treated with small molecules that have the same target in the cell, the alteration of target proteins should be similar in the cell. Proteome analysis by two-dimensional fluorescence differential gel electrophoresis (2D-DIGE) is an effective method for detecting the alternation of proteins in small molecule-treated cells. Comparison of protein expression patterns between cells that are treated with a small molecule and cells that are treated with known inhibitors will lead to the identification of molecular targets of new small molecules. The proteome database of cells that are treated with authentic inhibitors will be useful for this analysis. To establish the basis of this database, we performed proteome analysis of HeLa cells treated with 18 well-known inhibitors by 2D-DIGE. As a result, inhibitors that shared the same target were categorized in the same cluster. Our results indicate that proteome profiling on small molecule-treated cells is a useful methodology for target prediction of small molecules.

\subsection{DETECTION OF BINDING BETWEEN PROTEINS AND SMALL MOLECULES}

Affinity matrices are very useful for the identification of ligand-binding proteins from cell lysates. There have been many reports on matrices, such as agarose, polyacrylamide, and so on. However, nonspecific binding of proteins to solid supports has often caused trouble in identifying specific target proteins. To solve the problem, Handa et al. devised new matrices to reduce nonspecific interactions with proteins (Chapter 2). The newly developed matrices are very effective in identifying target proteins. Kanoh et al. describe the conjugation technique of small molecules to matrices by using photoaffinity linkers [22]. Their approach depends on the reactivity of carbene species that are generated from trifluoromethylaryldiazirine on ultraviolet irradiation. It was demonstrated in model experiments that photo-generated carbenes were able to react with every small molecule that was tested and to produce multiple conjugates in most cases. It was also found in on-array immobilization experiments that various small molecules were immobilized, retaining their ability to interact with their target proteins. With this approach, photo-cross-linked chemical arrays of about 15,000 compounds were constructed. These chemical arrays are very useful not only for ligand 
screening but also for the study of structure-activity relationships of ligands and their target proteins. Previously, if one wanted to make affinity matrices, he or she had to investigate the structure-activity relationship (SAR). Now, an SAR study can be done on a chemical array that is immobilized with a series of derivatives by combining them with a fluorescently labeled protein [22]. When the target protein is applied onto the chemical array, we can detect binding between the ligand and the protein as a fluorescent spot. Because this immobilization method does not require any specific chemical structures, the compounds that are immobilized retain their binding ability to the protein targets. Chemical arrays have enabled the rapid detection of such ligand-protein interactions in a high-throughput manner, although a label on the protein is needed to observe these interactions. By combining surface plasmon resonance (SPR) imaging technology with the chemical array platform, we developed a novel platform that allows in situ observation of interactions between photo-linked chemicals on gold surfaces and unlabeled proteins in solution [23]. In synthesizing suitable photo-cross-linkers for SPR imaging, the interactions between small molecules and unlabeled proteins can be observed with a high $S / N$ ratio.

\subsection{TRENDS IN CHEMICAL BIOLOGY}

There have been many reports on bioactive natural products. Because some of them are promising candidates for medicinal purposes, it is necessary to identify their target molecules in cells. Otherwise, the small molecules may show severe side effects. On the contrary, even if a small molecule has no worth for medicinal use, it can potentially be useful as bioprobe when it has a specific molecular target in the cell. For example, lactacystin was isolated from a Streptomyces sp. as a differentiation inducer of neuroblastoma cells [24]. Its target molecule has been identified to be a subunit of the $20 \mathrm{~S}$ proteasome [25]. Now, lactacystin is used in a wide range of studies to reveal the biological roles of proteasome function in mammalian cells. Moreover, velcade, another proteasome inhibitor, was developed as an effective medicine for multiple myeloma [26]. Investigation on the properties of bioactive small molecules is a starting point in chemical biology research. New bioprobes will be characterized by our efforts, and some of them may be golden eggs of medicine to be developed by pharmaceutical industries.

\section{REFERENCES}

1. Osada, H. (1998). Bioprobes for investigating mammalian cell cycle control. $J$. Antibiot., 51, 973-981.

2. Osada, H. (2000). Trends in bioprobe research. In Bioprobes, H. Osada, ed. SpringerVerlag, Tokyo, pp. 1-14.

3. Schreiber, S. L. (1991). Chemistry and biology of immunophilins and their immunosuppressive ligands. Science, 251, 283-287. 
4. Schreiber, S. L., Crabtree, G. R. (1992). The mechanism of action of cyclosporin A and FK506. Immunol. Today, 13, 136-142.

5. Cliptone, N. A., Crabtree, R. C. (1992). Identification of calcineurin as a key signalling enzyme in T-lymphocyte activation. Nature, 357, 695-697.

6. Schreiber, S. L., Liu, J., Albers, M. W., et al. (1992). Molecular recognition of immunophilins and immunophilin-ligand complexes. Tetrahedron, 48, 2545-2558.

7. Dedera, D., Hu, W., Vander Heyden, N., et al. (1989). Viral protein R of human immunodeficiency virus types 1 and 2 is dispensable for replication and cytopathogenicity in lymphoid cells. J. Virol., 63, 3205-3208.

8. Cohen, E. A., Dehni, G., Sodroski, J. G., et al. (1990). Human immunodeficiency virus Vpr product is a virion-associated regulatory protein. J. Virol., 64, 3097-3099.

9. Watanabe, N., Nishihara, Y., Yamaguchi, T., et al. (2006). Fumagillin suppresses HIV-1 infection of macrophages through the inhibition of Vpr activity. FEBS Lett., 580, 2598-2602.

10. Ingber, D., Fujita, T., Kishimoto, S., et al. (1990). Synthetic analogues of fumagillin that inhibit angiogenesis and suppress tumour growth. Nature, 348 .

11. Teitelbaum, S. L. (2000). Bone resorption by osteoclasts. Science, 289, 1504-1508.

12. Osada, H. (2000). Differentiation. In Bioprobes, H. Osada, ed. Springer-Verlag, Tokyo, pp. 43-65.

13. Kawatani, M., Okumura, H., Honda, K., et al. (2008). The identification of an inhibition through the osteoclastogenesis via inhibition of glyoxalase I. Proc. Natl. Acad. Sci. USA, 105, 11691-11696.

14. Hovius, R., Schmid, E. L., Tairi, A. P., et al. (1999). Fluorescence techniques for fundamental and applied studies of membrane protein receptors: the 5-HT3 serotonin receptor. J. Recept. Signal Transduct. Res., 19, 533-545.

15. Simizu, S., Osada, H. (2000). Mutations in the Plk gene lead to instability of Plk protein in human tumor cell lines. Nat. Cell Biol., 2, 852-854.

16. Watanabe, N., Sekine, T., Takagi, M., et al. (2009). Deficiency in chromosome congression by the inhibition of Plk1 polo box domain-dependent recognition. J. Biol. Chem., 284, 2344-2353.

17. Inamori, Y., Muro, C., Sajima, E., et al. (1997). Biological activity of purpurogallin. Biosci. Biotechnol. Biochem., 61, 890-892.

18. Kitada, S., Leone, M., Sareth, S., et al. (2003). Discovery, characterization, and structure-activity relationships studies of proapoptotic polyphenols targeting B-cell lymphocyte/leukemia-2 proteins. J. Med. Chem., 46, 4259-4264.

19. Stoughton, R. B., Friend, S. H. (2005). How molecular profiling could revolutionize drug discovery. Nat. Rev. Drug Discov., 4, 345-350.

20. Terstappen, G. C., Schlupen, C., Raggiaschi, R., et al. (2007). Target deconvolution strategies in drug discovery. Nat. Rev. Drug Discov., 6, 891-903.

21. Yaguchi, S., Fukui, Y., Koshimizu, I., et al. (2006). Antitumor activity of ZSTK474, a new phosphatidylinositol 3-kinase inhibitor. J. Natl. Cancer Inst., 98, 545-556.

22. Kanoh, N., Nakamura, T., Honda, K., et al. (2008). Distribution of photo-cross-linked products from 3-aryl-3-trifluoromethyldiazirines and alcohols. Tetrahedron, 64, 5692-5698. 
23. Saito, A., Kawai, K., Takayama, H., et al. (2008). Improvement of photoaffinity SPR imaging platform and determination of binding site of p62/SQSTM1 to p38 MAP kinase. Chem. Asian J., 3, 1607-1612.

24. Katagiri, M., Hayashi, M., Matsuzaki, K., et al. (1995). The neuritogenesis inducer lactacystin arrests cell cycle at both G0/G1 and G2 phases in neuro 2a cells. J Antibiot., 48, 344-346.

25. Fenteany, G., Standaert, R. F., Lane, W. S., et al. (1995). Inhibition of proteasome activities and subunit-specific amino-terminal threonine modification by lactacystin. Science, 268, 726-731.

26. Adams, J., (2004). The proteasome: a suitable antineoplastic target. Nat. Rev. Cancer, $4,349-360$. 